\title{
Glutaric Aciduria Type I Diagnosis Case with Normal Glutaryl Carnitine and Urine Organic Acid Analysis
}

\author{
(1) Ebru Canda1, (1) Havva Yazıcı1, (1) Esra Er1, (1) Cenk Eraslan2, (1) Yasemin Atik Altınok1, \\ (1) Hepsen Mine Serin3, (1) Sara Habif4, (1) Gül Serdaroğlu3, (D Sema Kalkan Uçar1, \\ (D) Hüseyin Onay5, (1) Ferda Özkınay5, (1) Mahmut Çoker1 \\ ${ }^{1}$ Ege University Faculty of Medicine, Department of Pediatrics, Division of Pediatric Metabolism and Nutrition, Izmir, Turkey \\ 2Ege University Facuty of Medicine, Department of Radiology, Izmir, Turkey \\ ${ }^{3}$ Ege University Faculty of Medicine, Department of Pediatrics, Division of Pediatric Neurology, Izmir, Turkey \\ 4Ege University Faculty of Medicine, Department of Biochemistry, Izmir, Turkey \\ ${ }^{5}$ Ege University Faculty of Medicine, Department of Genetics, Izmir, Turkey
}

\begin{abstract}
Glutaric aciduria Type I (GA-I) is a rare inherited metabolic disease, deficiency of glutaryl-CoA dehydrogenase results in accumulation of the putatively neurotoxic metabolites glutaric and 3-hydroxyglutaric acid (GA, 3-OH-GA) in body tissues, particularly within the brain. Here we presented a 3-year-old girl with hypotonia and dystonia diagnosed with GA-I although the repeated analysis of the carnitine profile and organic acid analyses were normal. The patient has motor, mental retardation, hypotonia. Her weight standard deviation score (SDS) was -1.86 SDS, height SDS was -0.55 SDS, head circumference SDS was -1.01. The physical examination was normal except severe hypotonia. Spot blood carnitine profile, blood amino acid, urine organic acid, lactic acid and pyruvic acid were normal in repeated analysis. Dystonia and spastic tetraparesis developed on her follow-up. Cranial magnetic resonance imaging revealed bilateral cortical atrophy and bilateral striatal and caudate nucleus $T 2$ flair hyperintensities. In GCDH gene analysis p.Y123C (c.368A>G)/p.L340F (c.368A $>$ G) mutation was found. There was no history of encephalopathy. The patient treated with levodopa and trihexyphenidyl and lysine-restricted diet. In the presence of bilateral striatal involvement and cortical atrophy and dystonia, GA-I should be kept in mind. Blood carnitine profile and urine organic acid analyses may not be consistent. It is important to evaluate the cases for genetic investigation.

Keywords: Glutaric acid, glutaryl carnitine, dystonia
\end{abstract}

\section{Introduction}

Glutaric aciduria Type I (GA-I, OMIM 231669) is a rare metabolic disorder of autosomal recessive inheritance caused by deficient or nonfunctional glutaryl-coenzyme A (CoA) dehydrogenase (GCDH). The GCDH gene is localized on chromosome 19p13.2 and encodes the protein that is involved in the catabolism of L-lysine, L-hydroxylysine and L-tyrptophan $(1,2)$. Acute neurological deterioration occurs most frequently between 6 and 18 months usually triggered by febrile illnesses or immunization. Movement disorder, dystonia, seizures and extrapyramidal symptoms are presented $(3,4)$. In few patients, neurological disease has been demonstrated in the absence of any encephalopathic crisis termed as insidious-onset type (5) and late onset type (6). Biochemically GCDH deficiency is characterized by a deposition of glutaric acid (GA, 3 hydroxyglutaric acid (3-OHGA), glutaconic acid (less frequently) and glutaryl carnitine (C5DC) (7). The major therapeutic principles GA-I are; the reduction of glutaryl CoA, GA, 3-OH-GA using low lysine diet and prevention of secondary carnitine deficiency (5). Here we presented a 4-year-old girl with hypotonia and dystonia and diagnosed with GA-I although the repeated analysis of the carnitine profile and organic acid analyses were normal. 


\section{Case Report}

Four years old girl was admitted to our clinic due to motor, mental retardation and hypotonia at the age of one year. She was born via vaginal delivery at $39^{\text {th }}$ gestational week, with $2900 \mathrm{~g}$ birth weight as the first child of nonconsanguineios parents. On her first pediatric neurology visit at the age of 11 months' global developmental delay was detected. Her weight was $7.2 \mathrm{~kg}$ [-1.86 standard deviation score (SDS)] and height was $72 \mathrm{~cm}(-0.55$ SDS) and head circumstance was 44 $\mathrm{cm}(-1.01 \mathrm{SDS})$. She was treated with physical therapy. When she was 18 months old, dystonia was detected. On physical examination severe hypotonia, and dystonia were detected, deep brain tendon reflexes were increased, and other systems were normal. Her eye examination was normal, and there was no hearing loss. There was no history of encephalopathy. Complete blood count results were in normal range. Renal function tests, liver function tests and creatine kinase levels were also normal. Spot blood carnitine profile, blood amino acid, urine organic acid, lactic acid and pyruvic acid were normal in repeated analyses. Cranial magnetic resonance imaging (MRI) revealed bilateral cortical atrophy and bilateral striatal and caudate nucleus T2 flair hyperintensities (Figure 1). Electromyogram analysis was normal. Feeding difficulties, hyper salivation and spastic tetra paresis developed on her follow-up. Cerebrospinal fluid (CSF) analysis performed and CSF glycine $79.1 \mu \mathrm{mol} / \mathrm{L}$, blood glycine $379.9 \mu \mathrm{mol} / \mathrm{L}$ and CSF glycine/blood glycine ratio was high (0.2). CSF serine, blood serine, CSF lactate and blood lactate were all normal. Due to the suspicion of non ketotic hyperglycinemia (NKH), Illumina Inherited Disease Sequencing Panel analysis performed and it was negative for NKH genes (glycine decarboxylase-GLDC, aminomethyltransferase, glycine cleavage system $\mathrm{H}$ proteinGCSH genes). In GCDH gene analysis p.Y123C (c.368A>G)/p. L340F (c.368A>G) mutation was found (Figure 2). Her mother has heterozygous p.L340F (c.368A $>\mathrm{G}$ ) mutation and her father has heterozygous p.Y123C (c.368A>G) mutation. After she had the diagnoses with GA-I, we repeated the CSF analysis and determined the normal levels of CSF and also blood glycine levels. The patient was treated with levodopa and trihexyphenidyl and lysine-restricted diet. Baclofen and diazepam treatment also given during follow up. Her last visit was at the age of 45 months. Failure to thrive was detected. Her weight was $9.6 \mathrm{~kg}(-2.78 \mathrm{SDS})$, her height was $89 \mathrm{~cm}$ (-4.6 SDS) and head circumstance $47 \mathrm{~cm}$ (-2.32 SDS). Spastic tetra paresis and dystonia was detected. During follow up all urine organic acid analyses were normal. Only in one sample spot blood glutaryl carnitine level was $0.49 \mu \mathrm{mol} / \mathrm{L}$ $(n=0-0.35)$ slightly elevated. We also detected high levels of creatine kinase levels (1422 IU/L; $n=34-145)$ which was returned to normal levels during follow up. During follow up clinical progression was observed and control cranial MRI investigation was performed. Findings were similar when compared with previous cranial MR.

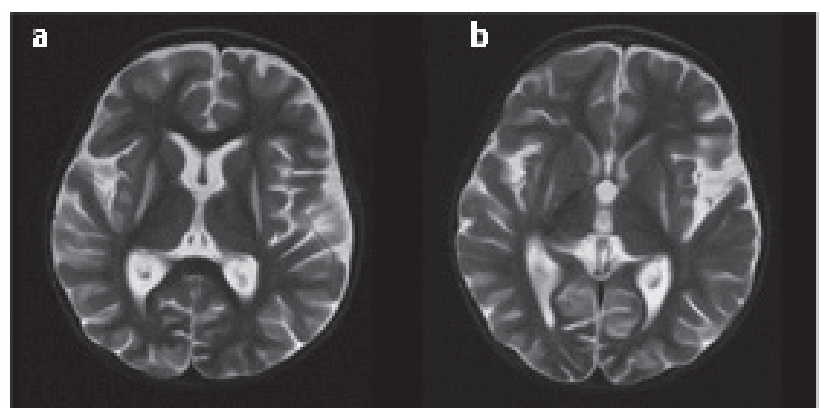

Figure 1. a, b) Axial T2, flair images show bilateral temporal atrophy and bilateral striatal hyperintensities

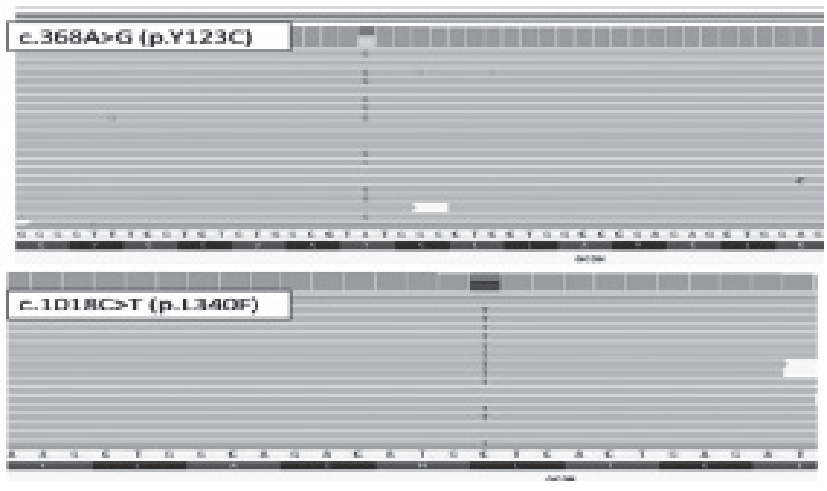

Figure 2. Molecular analysis of the patient

\section{Discussion}

GA-I is a rare neurometabolic disease and estimated prevalence is $1: 110000$ newborns (8). For most patients with GA-I, the acute encephalopathic crisis occurs and usually triggered by an infection or vaccination $(3,4)$. Recent studies suggest that neurological damage may occur in the absence of encephalopathic crisis and has been termed insidiousonset (9). In our patient there was no history of acute encephalopathic crisis, but progressive neurodegeneration was detected. Macrocephaly is found $75 \%$ of the patients with GA-I, but is non-specific (9) Also macrocephaly was absent in our patient. In the literature it was shown that epilepsy is frequent in patients with GA-I (8) unlikely our patient had no seizure. Like to the literature dystonia was the dominant extrapyramidal symptom in our patient (9). CSF analysis was performed in our patient due to presence of dystonia. High CSF glycine level and high CSF glycine/ plasma glycine ratio was detected. Movement disorders may present in late onset NKH patients (10). But in our patient's dystonia was started at 18 months at an early age and control CSF glycine levels detected within normal range. We could not find any explanation for this issue. She had no seizure and no treatment when CSF sample was taken. So we ruled out $\mathrm{NKH}$ by genetic analysis. Urine organic acid analysis usually shows increased levels of GA, 3-OH-GA, glutaryl-CoA, glutarylcarnitine although GA and dicarboxylic carnitines can be completely normal in some patients (11). In GA-l; brain GA has been found to exceed plasma and CSF 
levels even in patients with normal or low GA levels in urine, best explained by Kölker et al (3). It has been shown that urine $\mathrm{C} 5 \mathrm{DC}, \mathrm{GA}$ and $3-\mathrm{OH}-\mathrm{GA}$ did not show any correlation with the patients' clinics $(3,12)$. We had not performed urine C5DC, but all urine organic acid analyses were normal during follow up. Only one spot blood carnitine analysis, slightly high glutaryl carnitine level was detected. We want to mention that normal blood carnitine and urine organic acid analysis can be found in patients with GA-I. Cranial MRI plays an important role in the diagnostic work-up in GA-I patients. Many characteristic findings have been reported focusing on striatal changes, subdural collection and widening Sylvian fissures, abnormal signal intensity of caudate and putamen. Abnormalities of other gray matter structures have been rarely reported (12). The MRI findings of our patient such as striatal hyperintensities and temporal atrophy are common in GA-I patients. Our patient had the diagnosed by Illumina Inherited Disease Sequencing Panel analysis. GCDH gene analysis revealed p.Y123C (c.368A>G) mutation on one allele and p.L340F (c.1018C>T) mutation on the other allele. To the best of our knowledge this mutation has not been published in the literature.

Basic metabolic treatment of $\mathrm{GA}-\mathrm{l}$ is using low lysine diet for reduction of glutaryl-CoA, GA and 3-OH-GA and carnitine supplementation to prevent carnitine depletion. Riboflavin treatment is less frequent (5). Carnitine status and plasma amino acid concentrations should be monitored in GA-I patients (9). The value of metabolic treatment is unclear in patients with severe neurological deterioration, however it may be beneficial to the progression of the symptoms (9). Dietary treatment in combination with carnitine and emergency treatment has been shown to be effective in preventing neurological disease (5). We treated our patient with low lysine diet and carnitine supplementation. During follow up, spot blood free carnitine levels of the patient were normal. Also, repeated urine organic acid analyses were all normal. With the basic metabolic treatment, we didn't detect progression of the patients' symptoms but also no beneficial effect. GA-I is a treatable inborn metabolic disorder. It is important including GA-I in the differential diagnosis of leukoencephalopathy, dystonia combined with macrocephaly or not. The encephalopathic crisis may be absent and glutaryl carnitine elevation and urine glutaric acid elevation can not be detected. The diagnosis should be suspected on the combination of clinical and neuroradiological findings of the patient and GCDH gene analysis should be performed in such cases.

\section{Ethics}

Informed Consent: Informed concent form was filed out by all participants.
Peer-review: External and internal peer-reviewed.

\section{Authorship Contributions}

Surgical and Medical Practices: E.C., M.Ç., Concept: E.C., Y.A.A., Design: M.Ç., S.K.U., Data Collection or Processing: H.M.S., G.S., C.E., Analysis or Interpretation: H.O., FÖ., S.H., Literature Search: H.Y., E.E. Writing: E.C., S.K.U.

Conflict of Interest: No conflict of interest was declared by the authors.

Financial Disclosure: The authors declared that this study received no financial support.

\section{References}

1. Goodman SI, Markey SP, Moe PG, Miles BS, Teng CC. Glutaric aciduria; a "new" disorder of amino acid metabolism. Biochem Med 1975;12:12-21.

2. Koeller DM, DiGiulio KA, Angeloni SV, et al. Cloning, structure, and chromosome localization of the mouse glutaryl-CoA dehydrogenase gene. Genomics 1995;28:50812.

3. Kölker S, Garbade SF, Greenberg CR, et al. Natural history, outcome, and treatment efficacy in children and adults with glutaryl-CoA dehydrogenase deficiency. Pediatr Res 2006;59:840-7.

4. Bjugstad KB, Goodman SI, Freed CR. Age at symptom onset predicts severity of motor impairment and clinical outcome of glutaric acidemia type 1. J Pediatr 2000;137:681-6.

5. Kölker S, Christensen E, Leonard JV, et al. Guideline for the diagnosis and management of glutaryl-CoA dehydrogenase deficiency (glutaric aciduria type I). J Inherit Metab Dis 2007;30:5-22.

6. Külkens S, Harting I, Sauer S, et al. Late-onset neurologic disease in glutaryl-CoA dehydrogenase deficiency. Neurology 2005;64:2142-4

7. Baric I, Wagner L, Feyh P, Liesert M, Buckel W, Hoffmann GF. Sensitivity and specificity of free and total glutaric acid and 3-hydroxyglutaric acid measurements by stable-isotope dilution assays for the diagnosis of glutaric aciduria type I. J Inherit Metab Dis 1999;22:867-81.

8. Boy N, Mühlhausen C, Maier EM, et al. Proposed recommendations for diagnosing and managing individuals with glutaric aciduria type I: second revision. J Inherit Metab Dis 2017;40:75-101.

9. Kölker S, Christensen E, Leonard JV, et al. Diagnosis and management of glutaric aciduria type l--revised recommendations. J Inherit Metab Dis 2011;34:677-94.

10. Lee WT. Disorders of amino acid metabolism associated with epilepsy. Brain Dev 2011;33:745-52.

11. Pineda M, Ribes A, Busquets $C$, Vilaseca MA, Aracil A, Christensen E. Glutaric aciduria type I with high residual glutaryl-CoA dehydrogenase activity. Dev Med Child Neurol 1998;40:840-2.

12. Couce ML, López-Suárez $\mathrm{O}$, Bóveda $M D$, et al. Glutaric aciduria type I: outcome of patients with early- versus latediagnosis. Eur J Paediatr Neurol 2013;17:383-9. 